\title{
Correlation between Mineral Intake and Urinary Excretion in Free-Living Japanese Young Women
}

\author{
Munehiro Yoshida $^{{ }^{*}}$, Tsutomu Fukuwatari ${ }^{2}$, Junya Sakai ${ }^{2}$, Tomiko Tsuji ${ }^{2,3}$, Katsumi Shibata ${ }^{2}$ \\ ${ }^{1}$ Laboratory of Food and Nutritional Sciences, Faculty of Chemistry, Materials and Bioengineering, Kansai University, Suita, Japan; \\ ${ }^{2}$ Laboratory of Food Science and Nutrition, Department of Lifestyle Studies, School of Human Cultures, The University of Shiga \\ Prefecture, Hikone, Japan; ${ }^{3}$ Department of Health and Nutrition, School of Health and Human Life, Nagoya Bunri University, Ina- \\ zawa, Japan. \\ Email: *hanmyou4@kansai-u.ac.jp
}

Received October $8^{\text {th }}, 2011$; revised November $8^{\text {th }}, 2011$; accepted November $25^{\text {th }}, 2011$

\begin{abstract}
To clarify whether the urinary excretion of calcium, magnesium, phosphorus, iron, zinc, copper, manganese, selenium and molybdenum can be used as an index of their intake, the association between urinary excretion and intake in free-living individuals was examined. A total of 102 healthy free-living female university dietetics students aged 18 - 33 years voluntarily participated in this study, of which 76 students were eligible for this assessment. All food consumed for four consecutive days was recorded accurately by a weighed food record method. A 24-h urine sample was collected on the fourth day, and the urinary levels of sodium, potassium, calcium, magnesium, phosphorus, iron, zinc, copper, manganese, selenium and molybdenum were measured. Significant correlation between urinary excretion and intake was observed in sodium $(r=0.596, p<0.001)$, potassium $(r=0.583, p<0.001)$, calcium $(r=0.402, p<0.001)$, magnesium ( $r=0.365, p<0.01)$, phosphorus $(r=0.509, p<0.001)$, selenium $(r=0.349, p<0.01)$ and molybdenum $(r=$ $0.265, p<0.01)$. On the other hand, urinary excretion was very low and completely independent of the intake in iron, zinc, copper and manganese. These results indicate that urinary calcium, magnesium, phosphorus, selenium and molybdenum can be used as an index of their intake, similarly to sodium and potassium.
\end{abstract}

Keywords: Mineral Intake; Trace Elements; Urinary Excretion; Assessment; Japanese Young Women

\section{Introduction}

To assess the nutritional status of healthy free-living humans, the weighed food record method has been used widely to record the dietary intake and to calculate nutrient intake [1]. Although this method can provide relatively precise information regarding dietary intake compared with other dietary assessment [2], substantial effort is required for respondents to complete the dietary records and to weigh all food consumed. This often leads to errors in the records, which reveals the limitation of a weighed food record method in terms of accuracy [3]. Alternatively, other methods using quantitative biological information, such as urinary excretion, or concentrations of nutrient or their metabolites in blood, as biomarkers to assess dietary intake or nutritional status have been well studied in recent years.

Many preceding studies have investigated urinary excretion as a biomarker for assessing dietary intake. For example, 24-h urinary nitrogen is established as a marker for protein intake [4], urinary sugars for sugar intake ${ }^{*}$ Corresponding author.
[5,6], and urinary thiamine for thiamine intake [7]. As regards minerals, urinary potassium is established as a marker for potassium intake [8] and urinary iodine for iodine intake [9] as well as urinary sodium for sodium intake [10,11].

In the present study, we measured sodium, potassium, calcium, magnesium, phosphorus, iron, zinc, copper, manganese, selenium and molybdenum in 24-h urine and examined the association between urinary mineral excretion and their intake in free-living individuals. In addition, we examined whether the urinary excretion of calcium, magnesium, phosphorus, iron, zinc, copper, manganese, selenium and molybdenum can be used as an index of their intake, similarly to sodium and potassium.

\section{Subjects and Methods}

\subsection{Subjects}

This study was reviewed and approved by the Ethics Committee of The University of Shiga Prefecture. A total of 102 healthy free-living female university dietetics students aged 18 - 33 years voluntarily participated in this 
study. The purpose and protocol of this study was explained to all participants before joining the study, and written informed consent was obtained from each participant, and from parents of participants aged $<20$ years. We excluded participants diagnosed with cold or influenza, and those who had taken mineral supplements at least once during the previous month. In addition, we excluded participants whose 24-h urine collection or dietary records were considered as incomplete, with a collection time outside the 22 - $26 \mathrm{~h}$ range, urine volume < $250 \mathrm{~mL}$, creatinine excretion in relation to body weight outside the $10.8-25.2 \mathrm{mg} / \mathrm{kg}$ range [12], or extremely low or high energy intake $(<500$ or $>4000 \mathrm{kcal} / \mathrm{d})$. After screening, 76 participants were found to be eligible. Anthropometric profiles of the 76 participants are shown and compared with those of general Japanese young women in Table 1. No difference was observed between subjects and general women.

\subsection{Dietary Records}

This was a 4-day dietary assessment in which the participants were living freely at college and consuming their normal diet. The first day (Monday) of the experimental period was defined as Day 1, etc. To measure dietary intake during the 4-day period precisely, we used a weighed food record method, which is the highest quality in Japan at this time [13,14]. A digital cooking scale (1 g unit; Tanita Inc., Tokyo, Japan), a set of dietary record forms, a dietary record manual, and a disposable camera were distributed to the participants in advance. Upon entry of the dietary record, the status of food at oral intake was identified as "raw", "cooked", "the presence of skin", "cooking ingredient", or "with or without seasoning", and coded according to the Fifth Revised and Enlarged Edition of the Standard Tables of Food Composition in Japan [15]. The participants took photographs with a disposable camera of the dish before and after eating. Several experienced dietitians used the photographs to complete the data, and asked the participants to resolve any discrepancies or to obtain further information when needed. The food that remained after eating was measured by a digital scale and was deduced from the dietary record. Food, nutrient and energy intake was calculated using the Standard Tables of Food Composition

Table 1. Comparison of anthropometric profiles between subjects and general Japanese young women.

\begin{tabular}{lcc}
\hline & Subjects $(n=76)$ & NHNSJ-2008 $^{1}(n=284)$ \\
\hline Age & $20.1 \pm 2.3$ & $20-29$ \\
Height $(\mathrm{cm})$ & $158.3 \pm 5.0$ & $158.3 \pm 5.4$ \\
Weight $(\mathrm{kg})$ & $50.8 \pm 5.2$ & $51.9 \pm 9.5$ \\
Body mass index $\left(\mathrm{kg} / \mathrm{m}^{2}\right)$ & $20.2 \pm 1.7$ & $20.7 \pm 3.6$ \\
\hline
\end{tabular}

Values are the means $\pm \mathrm{SD} .{ }^{1}$ Values for general Japanese young women aged 20 to 29 years described in the National Health and Nutrition Survey of Japan in 2008. in Japan. For mineral intake, sodium, potassium, calcium, phosphorus, iron, zinc, copper and manganese were assessed. Because selenium and molybdenum are not designated in the Standard Table of Food Composition in Japan, intake of these microminerals was calculated using averaged values of the contents for every food groups described in the literature $[16,17]$.

\subsection{4-h Urine Sampling}

A single 24-h urine sample was collected on Day 4 to measure urinary mineral excretion. In the morning, participants were asked to discard the first specimen and to record the time on the sheet. The next morning, participants were asked to collect the last specimen at the same time as when the specimen had been discarded the previous morning, and to record the time on the sheet. After the urine sample had been collected, the volume of the sample was measured. The urine samples were stored at $-20^{\circ} \mathrm{C}$ until analysis.

\subsection{Measurement of Urinary Minerals}

Urine samples were diluted with 9 or more volumes of $0.1 \mathrm{M} \mathrm{HNO}_{3}$ and filtrated through a $0.45-\mu \mathrm{m}$-membrane filter. Filtrate thus obtained was used for the measurement of minerals. Sodium, potassium, calcium and magnesium were determined by atomic absorption spectrometer (AA-6300; Shimadzu, Kyoto, Japan). Phosphorus, iron, zinc and copper were determined by inductively coupled plasma-atomic emission spectrometer (ULTIMA2; Horiba Ltd., Kyoto, Japan). Manganese, selenium and molybdenum were determined by inductively coupled plasma-mass spectrometer (ICPM-8500; Shimadzu) using rhodium (for manganese and molybdenum) and tellurium (for selenium) as internal standards. In these urinalyses, recovery of each mineral adding urine was $97 \%$ to $101 \%$.

\subsection{Statistical Analysis}

For each subject, means of daily nutrient and energy intake were calculated from the consecutive 4-day dietary records. The mean values of the subjects were calculated based on the resulting individual mean values. Pearson correlation coefficients were calculated to determine the association between urinary and dietary measurements of minerals. These statistical tests were performed using a personal computer (eMac; Apple Computer, Cupertino, CA, USA) with the operating system Mac OS 9.2 and statistical program package StatView-J version 5.0 (Abacus Concept, Berkeley, CA).

\section{Results and Discussion}

In Table 2, the daily energy and nutrient intake of the 76 
Table 2. Daily intake of energy, major nutrients and minerals of subjects at experimental period.

\begin{tabular}{lll}
\hline & $\begin{array}{l}\text { Subjects }^{1} \\
(n=76)\end{array}$ & $\begin{array}{l}\text { NHNSJ-2008 } \\
(n=418)\end{array}$ \\
\hline Energy (kcal) & $1658 \pm 302$ & $1669 \pm 475$ \\
Protein (g) & $57.3 \pm 11.9$ & $61.0 \pm 21.4$ \\
Lipid (g) & $52.8 \pm 15.5$ & $53.7 \pm 22.6$ \\
Carbohydrate (g) & $232.8 \pm 39.8$ & $227.3 \pm 66.6$ \\
Minerals & & \\
Sodium (mg) & $2923 \pm 834$ & $3617 \pm 1415^{3}$ \\
Potassium (mg) & $1873 \pm 472$ & $1886 \pm 710$ \\
Calcium (mg) & $503 \pm 142$ & $406 \pm 205^{3}$ \\
Magnesium (mg) & $194 \pm 53$ & $201 \pm 70$ \\
Phosphorus (mg) & $852 \pm 193$ & $844 \pm 292$ \\
Iron (mg) & $6.7 \pm 1.9$ & $6.7 \pm 2.7$ \\
Zinc (mg) & $6.9 \pm 1.5$ & $7.2 \pm 2.6$ \\
Copper (mg) & $0.90 \pm 0.21$ & $0.98 \pm 0.34$ \\
Manganese (mg) & $2.8 \pm 0.8$ & - \\
Selenium ( $\mu$ g) & $189 \pm 67$ & - \\
Molybdenum $(\mu g)$ & $272 \pm 77$ & - \\
\hline
\end{tabular}

Values are the means $\pm \mathrm{SD} .{ }^{1}$ Daily intake was assessed from the consecutive 4-day dietary records. ${ }^{2}$ Values for general Japanese young women aged 18 to 29 years described in the National Health and Nutrition Survey of Japan in 2008. ${ }^{3}$ Significant difference was observed between subjects and general Japanese young women at $p<0.001$ by Student's $t$-test.

eligible participants is presented and compared with those of general Japanese young women described in the National Health and Nutritional Survey of Japan (NHNSJ) [18]. Similarity was observed between the subjects and general Japanese in the intake of energy and macronutrients. Among minerals, no difference was observed in potassium, magnesium, phosphorus, iron, zinc and copper intake. In addition, manganese and molybdenum intake in the participants was close to the reported values for general Japanese $[19,20]$. On the other hand, lower sodium intake and higher calcium intake were observed in the subjects than in general young women. In Japan, because excess intake of sodium and low intake of calcium have been major nutritional problems, dietetics students have received education so that sodium intake is reduced and calcium intake is increased; therefore, it is thought that the subjects made efforts to reduce their sodium intake and increase their calcium intake intentionally. Selenium intake in the participants was quite a bit higher than the reported value for general Japanese [16,21]. This indicates that overestimation arose in selenium intake roughly calculated using averaged values of the contents for every food group because no difference was observed between the subjects and general Japanese adolescents in the intake of energy and many nutrients.

Table 3 shows 24-h urinary excretion and the apparent urinary excretion rate of minerals. As regards manganese, since almost all samples showed less than the detection limit $(<10 \mu \mathrm{g} / \mathrm{L})$, it is excluded from the table.
Table 3. Daily urinary mineral excretion in subjects.

\begin{tabular}{lll}
\hline & Excretion amounts & Apparent excretion rate \\
\hline & $(\mathrm{mg} / \mathrm{d})$ & $(\%)$ \\
Sodium & $2616 \pm 1010$ & $90.7 \pm 30.8$ \\
Potassium & $1456 \pm 498$ & $79.5 \pm 23.0$ \\
Calcium & $100.5 \pm 36.4$ & $20.9 \pm 8.2$ \\
Magnesium & $39.9 \pm 16.4$ & $22.4 \pm 15.4$ \\
Phosphorus & $660 \pm 223$ & $79.1 \pm 23.8$ \\
& $(\mu \mathrm{g} / \mathrm{d})$ & \\
Iron & $220 \pm 138$ & $3.6 \pm 2.5$ \\
Zinc & $374 \pm 125$ & $6.3 \pm 2.8$ \\
Copper & $52.5 \pm 37.1$ & $6.3 \pm 5.1$ \\
Selenium & $84.8 \pm 26.6$ & $49.7 \pm 21.3$ \\
Molybdenum & $211 \pm 93$ & $82.2 \pm 44.3$ \\
\hline
\end{tabular}

Values are the means $\pm \mathrm{SD}$. Apparent excretion rate was calculated as follows: (daily urinary excretion amounts)/(daily intake) $\times 100$.

A high rate of urinary excretion ( $>70 \%)$ was observed for sodium and potassium, which intake has been assessed using urine. In addition, phosphorus and molybdenum also showed a high excretion rate, parallel to sodium and potassium. Because most phosphorus and molybdenum ingested from food are absorbed in the intestine and their main excretion route is urine [20,22], this high excretion rate is valid. Although dietary selenium is also mostly absorbed and its main excretion route is urine [23], the excretion rate was $50 \%$, which was lower than several reported values [24]. This was surely caused by an overestimation of selenium intake; if the excretion rate were $70 \%$, selenium intake would be estimated to be about $120 \mu \mathrm{g} / \mathrm{d}$, which is almost coincident with the reported value for general Japanese [16,21].

The apparent urinary excretion rate of calcium and magnesium was about $20 \%$, which was coincident with the reported value [22,25]. On the other hand, urinary excretion of iron, zinc and copper was very low, which reflects that urine is not the main excretion route of these minerals [26-28].

Figure 1 shows the correlation between daily intake and 24-h urinary excretion of sodium, potassium, calcium, magnesium and phosphorus. Significant correlation was observed with all of these five minerals. In particular, a strong correlation $(r>0.5)$ was observed for sodium, potassium and phosphorus; therefore, in these three minerals, intake could be estimated from the amount of urinary excretion for every individual with high accuracy. Urinary sodium and potassium are already used as important indices of their intake for individuals $[10,11]$. In addition, urinary phosphorus could also be used as an index of its intake.

Also, in the case of calcium and magnesium, a significant correlation between urinary excretion and intake was observed. The intestinal absorption rate of calcium and magnesium is $30 \%$ to $50 \%$ and the main excretion 

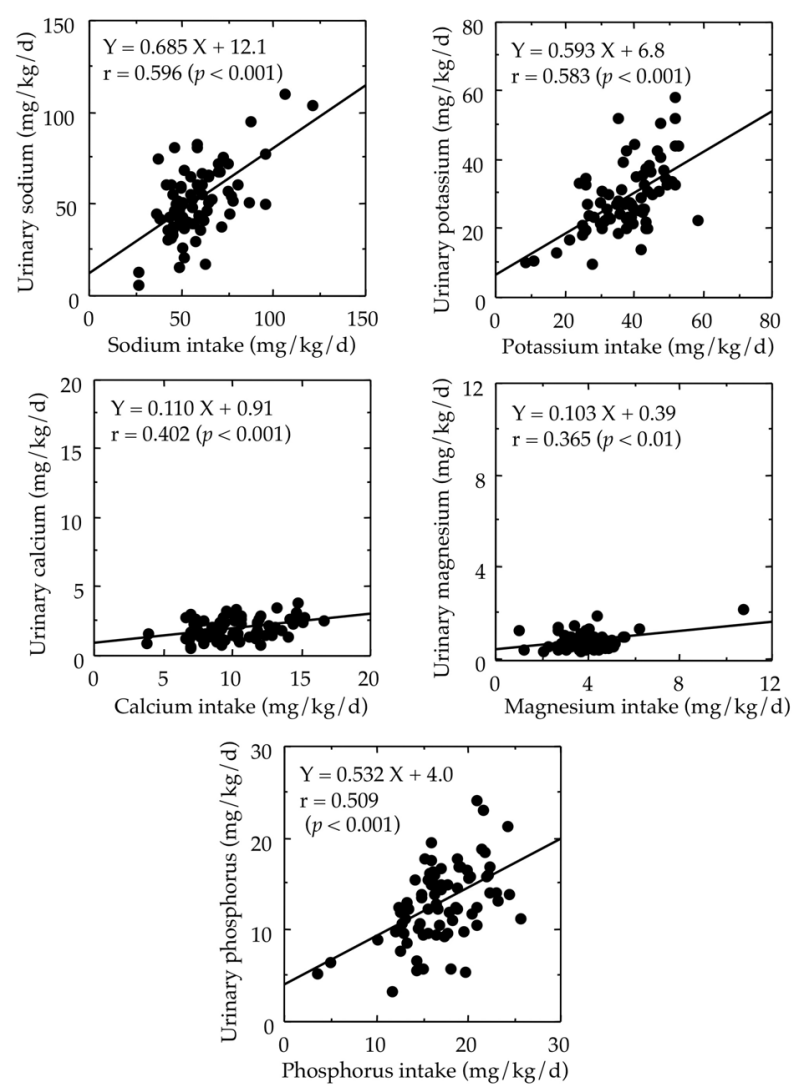

Figure 1. Correlation between daily intake and urinary excretion of sodium, potassium, calcium, magnesium and phosphorus in subjects.

route is urine [22,25]; therefore, urinary excretion of these minerals reflects absorption amounts. Since intestinal absorption of these minerals changes with various factors [29], it may be difficult to estimate the intake of these minerals from the urinary excretion for every individual. Nevertheless, it will be possible to estimate the intake from urinary excretion at least in a group.

Figure 2 shows correlation between intake and urinary excretion in iron, zinc, copper, selenium and molybdenum. In iron, zinc and copper, the scale is changed between the $\mathrm{X}$ - and $\mathrm{Y}$-axis since their excretion rate to urine is very low. In these three minerals, urinary excretion was almost completely independent of the intake. Accordingly, intake of these minerals cannot be estimated from urinary excretion. In addition, because urinary manganese excretion was very low, similarly to iron, zinc and copper, it may be difficult to use urinary manganese as an index of manganese intake. Probably, it is the reason that their urinary excretion is constantly low regardless of the intake, since they are bound to protein in blood. In the case of selenium and molybdenum, a significant correlation was observed; however, in spite of having said that a large part of ingested selenium and molybdenum was excreted into urine, similarly to potas
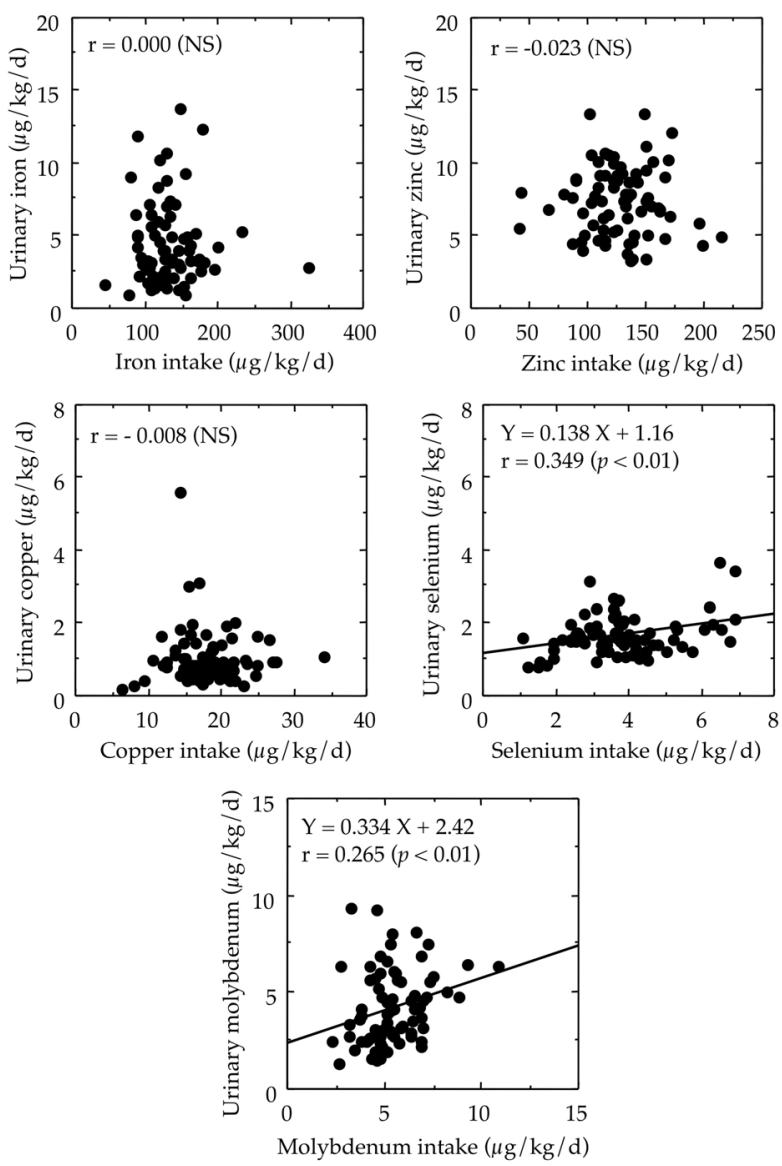

Figure 2. Correlation between intake and urinary excretion of iron, zinc, copper, selenium and molybdenum of subjects.

sium, sodium and phosphorus [20,23], the correlation coefficients were smaller than those of calcium and magnesium. Probably, these weak correlations were due to rough intake estimation using averaged values of the contents for every food group; therefore, it is considered that a greater correlation coefficient was obtained when intake was estimated using the content of every food, as for other minerals.

In the present study, it was confirmed that excretion amounts in 24-h urine were good indices of daily intake of phosphorus, calcium, magnesium, selenium and molybdenum similarly to sodium and potassium. In minerals, estimation of the intake using 24-h urine is possible when the main excretion route is urine. To estimate the intake of these minerals from the urinary excretion, the precise regression between intake and urinary excretion needs to be established by a balance test in the future.

\section{Acknowledgements}

This study was supported by a grant for comprehensive research on cardiovascular and lifestyle disease from the Ministry of Health, Labour and Welfare of Japan. 


\section{REFERENCES}

[1] W. Wilett, "Nutritional Epidemiology,” 2nd Edition, Oxford University Press, New York, 1998.

[2] S. A. Bingham, C. Gill, A. Welch, A. Cassidy, S. A. Runswick, S. Oakes, R. Lubin, D. I. Thurnham, T. J. Key, L. Roe, K. T. Khaw and N. E. Day, "Validation of Dietary Assessment Methods in the UK Arm of EPIC Using Weighed Records, and 24-Hour Urinary Nitrogen and Potassium and Serum Vitamin C and Carotenoids as Biomarkers,” International Journal of Epidemiology, Vol. 26, Suppl. 1, 1997, pp. S137-S151. doi:10.1093/ije/26.suppl_1.S137

[3] M. B. E. Libingstone and A. E. Black, "Markers of the Validity of Reported Energy Intake,” Journal of Nutrition, Vol. 133, Suppl. 3, 2003, pp. 895S-920S.

[4] S. A. Bingham, "Urine Nitrogen as a Biomarker for the Validation of Dietary Protein Intake," Journal of Nutrition, Vol. 133, Suppl. 3, 2003, pp. 921S-924S.

[5] C. Luceri, G. Caderni, M. Lodovici, M. T. Spagnesi, C. Monserrat, L. Lancioni and P. Dolara, "Urinary Excretion of Sucrose and Fructose as a Predictor of Sucrose Intake in Dietary Intervention Studies,” Cancer Epidemiology, Biomarkers and Prevention, Vol. 5, No. 3, 1996, pp. 167171.

[6] N. Tasevska, S. A. Runswick, A. A. Welch, A. Mc- Taggart and S. A. Bingham, "Urinary Sugars Biomarker Relates Better to Extrinsic than to Intrinsic Sugars Intake in a Metabolic Study with Volunteers Consuming Their Normal Diet," European Journal of Clinical Nutrition, Vol. 63, No. 5, 2009, pp. 653-659.

doi:10.1038/ejcn.2008.21

[7] N. Tasevska, S. A. Runswick, A. McTaggart and S. A. Bingham, "Twenty-Four-Hour Urinary Thiamine as a Biomarker for the Assessment of Thiamine Intake," European Journal of Clinical Nutrition, Vol. 62, No. 9, 2008, pp. 1139-1147. doi:10.1038/sj.ejcn.1602829

[8] N. Tasevska, S. A. Runswick and S. A. Bingham, "Urinary Potassium Is as Reliable as Urinary Nitrogen for Use as a Recovery Biomarker in Dietary Studies of Free Living Individuals,” Journal of Nutrition, Vol. 136, No. 5, 2006, pp. 1334-1340.

[9] D. Ristic-Medic, Z. Piskackova, L. Hooper, J. Ruprich, A. Casgrain, K. Ashton, M. Pavlovic and M. Glibetic, "Methods of Assessment of Iodine Status in Humans: A Systematic Review," American Journal of Clinical Nutrition, Vol. 89, No. 6, 2009, pp. 2052S-2069S. doi:10.3945/ ajcn.2009. 27230H

[10] L. K. Dahl, "Evidence for an Increased Intake of Sodium in Hypertension Based on Urinary Excretion of Sodium," Proceedings of Society of Experimental Biology and Medicine, Vol. 94, No. 1, 1957, pp. 23-26.

[11] Y. Morinaga, T. Tsuchihashi, Y. Ohta and K. Matsumura, "Salt Intake in 3-Year-Old Japanese Children," Hypertension Research, Vol. 34, No. 7, 2011, pp. 836-839. doi:10.1038/hr.2011.55

[12] K. Murakami, S. Sasaki, Y. Takahashi, K. Uenishi, T. Watanabe, T. Kohri, M. Yamasaki, R. Watanabe, K. Baba, K. Shibata, T. Takahashi, K. Hayabuchi, K. Ohki and J.
Suzuki, "Sensitivity and Specificity of Published Strategies Using Urinary Creatinine to Identify Incomplete 24-h Urine Collection,” Nutrition, Vol. 24, No. 1, 2008, pp. 16-22. doi:10.1016/j.nut.2007.09.001

[13] T. Imai, S. Sasaki, K. Mori, F. Ando, N. Niino and H. Shimokata, "Nutritional Assessment of 3-Day Dietary Records in National Institute for Longevity ScienceLongitudinal Study of Aging (NILS-LSA)," Journal of Epidemiology, Vol. 10, Suppl. 1, 2000, pp. S70-S76. doi:10.2188/jea.10.1sup 70

[14] K. Murakami, H. Okubo and S. Sasaki, "Dietary Intake in Relation to Self-Reported Constipation among Japanese Women Aged 18 - 20 Years,” European Journal of Clinical Nutrition, Vol. 60, No. 5, 2006, pp. 650-657. doi:10.1038/sj.ejcn.1602365

[15] Ministry of Education, Culture, Sport, Science and Technology of Japan, "Standard Tables of Food Composition in Japan 5th Revised and Enlarged Edition (in Japanese)," National Printing Bureau of Japan, Tokyo, 2007.

[16] Y. Miyazaki, H. Koyama, Y. Sasada, H. Sato, M. Nojiri and S. Suzuki, "Dietary Habits and Selenium Intake of Residents in Mountain and Coastal Communities in Japan,” Journal of Nutritional Science and Vitaminology, Vol. 50, No. 5, 2004, pp. 309-319. doi:10.3177/jnsv.50.309

[17] H. Hattori, A. Ashida, C. Itô and M. Yoshida, "Determination of Molybdenum in Foods and Human Milk, and an Estimate of Average Molybdenum Intake in the Japanese population,” Journal of Nutritional Science and Vitaminology, Vol. 50, No. 6, 2004, pp. 404-409. doi:10.3177/jnsv.50.404

[18] Ministry of Health, Labour and Welfare of Japan, “The National Health and Nutrition Survey in Japan, 2008 (in Japanese),” Dai-Ichi Shuppan, Tokyo, 2011.

[19] S. Horiguchi, K. Teramoto, T. Kurono and K. Ninomiya, "The Arsenic, Copper, Lead, Manganese and Zinc Contents of Daily Foods and Beverages in Japan and the Estimate of Their Daily Intake,” Osaka City Medical Journal, Vol. 24, No. 1, 1978, pp. 131-141.

[20] M. Yoshida, H. Hattori, S. Ota, K. Yoshihara, N. Kodama, Y. Yoshitake and M. Nishimuta, "Molybdenum Balance in Healthy Young Japanese Women," Journal of Trace Elements in Medicine and Biology, Vol. 20, No. 4, 2006, pp. 245-252. doi:10.1016/j.jtemb.2006.07.004

[21] M. Yoshida and K. Yasumoto, "Selenium Content of Rice Grown at Various Sites in Japan,” Journal of Food Composition and Analysis, Vol. 1, No. 1, 1987, pp. 71-75. doi:10.1016/0889-1575(87)90013-5

[22] M. Nishimuta, N. Kodama, E. Morikuni, Y. H. Yoshioka, H. Takeyama, H. Yamada, H. Kitajima and K. Suzuki, "Balances of Calcium, Magnesium and Phosphorus in Japanese Young Adults,” Journal of Nutritional Science and Vitaminology, Vol. 50, No. 1, 2004, pp. 19-25. doi:10.3177/jnsv.50.19

[23] C. A. Swanson, D. C. Reamer, C. Veillon, J. C. King and O. A. Levander, "Quantitative and Qualitative Aspects of Selenium Utilization in Pregnant and Nonpregnant Women: An Application of Stable Isotope Methodology,” American Journal of Clinical Nutrition, Vol. 38, No. 2, 
1983, pp. 169-180.

[24] H. J. Robbrecht and H. A. Deelstra, "Selenium in Human Urine: Concentration Levels and Medical Implications," Clinica Chimica Acta, Vol. 136, No. 2-3, 1984, pp. 107120. doi:10.1016/0009-8981(84)90282-1

[25] N. M. Lewis, M. S. Marcus, A. R. Behling and J. L. Greger, "Calcium Supplements and Milk: Effects on AcidBase Balance and on Retention of Calcium, Magnesium, and Phosphorus," American Journal of Clinical Nutrition, Vol. 49, No. 3, 1989, pp. 527-533.

[26] Food and Nutrition Board Institute of Medicine, "Iron," Dietary Reference Intakes for Vitamin A, Vitamin K, Arsenic, Boron, Chromium, Copper, Iodine, Iron, Manganese, Molybdenum, Nickel, Silicon, Vanadium, and Zinc,
National Academy Press, Washington DC, 2001, pp. 290393.

[27] J. R. Turnlund, “Copper,” In: M. E. Shils, J. A. Olson, M. Shike and A. C. Ross, Eds., Modern Nutrition in Health and Disease, 9th Edition, Wikkiams \& Wilkins, Baltimore, 1999, pp. 241-252.

[28] J. C. King and C. L. Keen, “Zinc,” In: M. E. Shils, J. A. Olson, M. Shike and A. C. Ross, Eds., Modern Nutrition in Health and Disease, 9th Edition, Wikkiams \& Wilkins, Baltimore, 1999, pp. 223-239.

[29] T. Hazell, "Minerals in Foods: Dietary Sources, Chemical Forms, Interactions, Bioavailability," World Review of Nutrition and Dietetics, Vol. 46, 1985, pp. 1-123. 